\title{
Análisis de programas de mejora continua. Un estudio longitudinal en una empresa industrial
}

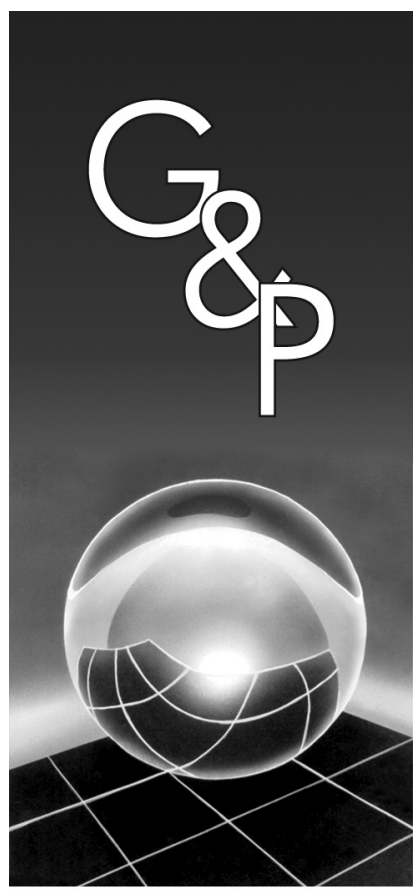

\author{
Juan A. Marin-Garcia \\ Manuela Pardo-del-Val \\ Tomas Bonavia
}

\begin{abstract}
Resumen
Las empresas han utilizado diversas herramientas que permiten que los operarios contribuyan al proceso de mejora continua. Entre las herramientas más usadas podemos destacar los sistemas de sugerencias tanto individuales como en grupo. En esta comunicación haremos un repaso de las principales características de ambos sistemas y los modos habituales de implantación. Nuestra ponencia pretende intentar responder a estas preguntas de investigación. ¿Qué resultados se derivan de la implantación de sistemas de sugerencias individuales o en grupo? ¿Cuál de los dos sistemas es más beneficioso para la empresa? ¿Qué problemas surgen durante el funcionamiento de estos programas? Para ello, analizaremos los datos de un caso de empresa industrial donde hemos recogido los datos históricos de 5 años de aplicación de un programa de mejora continua. Ambos programas han demostrado ser provechosos para la empresa, aunque las posibilidades de los sistemas de grupo parecen ser significativamente mayores.
\end{abstract}

Palabras clave: Mejora continua. Sistemas de sugerencias. Equipos de mejora. Resultados. Reducción de costes.

\section{Introducción}

El entorno actual en el que se mueven muchas de las empresas industriales está caracterizado por una fuerte competencia (BOND, 1999), con un papel cada vez más predominante de las nuevas tecnologías (GARCIALORENZO; PRADO, 2003). En este contexto, la mejora continua es una arma para mantener y mejorar la competitividad, aprovechando el conocimiento y la implicación de los obreros de la empresa (GARCIA-LORENZO; PRADO, 2003; PRADO PRADO, 1998; TERZIOVSKI; SOHAL, 2000; VAN DIJK; VAN DEN ENDE, 2002; WOOD, 2003). Aunque la mejora continua puede estar originada por las ideas o propuestas de mandos, técnicos o consultores, también puede ser animada por la creatividad e implicación de los obreros (BODEK, 2002; FAIRBANK; WILLIAMS, 2001; GARCIA-LORENZO; PRADO, 2003; GRÜTTER et al., 2002; KERRIN; OLIVER, 2002; LLOYD, 1999; PRADO, 2001). En este sentido, los sistemas de sugerencias y los equipos de mejora son algunas de las herramientas que permiten poner en marcha la mejora continua aprovechando las ideas de los obreros.

Múltiples autores han considerado la mejora continua como una de las herramientas básicas para la implan- tación de sistemas de producción basados en gestión de la calidad total, producción ajustada o empresas de primer nivel mundial (World Class Manufacturing) (BACDAYAN, 2001; BOND, 1999; FRESE et al., 1999; GRÜTTER et al., 2002; JACKSON; DYER, 1998; SCHONBERGER, 1996; TAPPING et al., 2002). Sin embargo, la mejora continua se puede utilizar como una herramienta para el funcionamiento de cualquier organización, aunque no se base en estas metodologías avanzadas (RAPP; EKLUND, 2002). No debemos olvidar la importancia que tiene para la empresa la implantación de pequeñas mejoras con carácter acumulativo, que acaban produciendo resultados importantes y duraderos (BOND, 1999; CHOI et al., 1997; FAIRBANK; WILLIAMS, 2001). Llama la atención que no hay demasiadas publicaciones en revistas académicas que se ocupen de la mejora continua, lo que contrasta con el hecho de estar en la base de muchas metodologías sobre las que se publica con mucha más frecuencia (FRESE et al., 1999). De hecho, la Continuous Innovation Network (CINet), organización que engloba a los investigadores que trabajan en este tema, en su afán por desarrollar y extender los resultados de las investigaciones sobre mejora continua ha optado 
por crear su propia publicación, "Research in Continuous Innovation".

Entre los principales motivos que tienen las empresas para implantar la mejora continua, podemos destacar la mejora de la productividad o la eficiencia (GRÜTTER et al., 2002; RAPP; EKLUND, 2002), la calidad (GRÜTTER et al., 2002), la reducción de costes de producción (BOND, 1999; MODARRESS et al., 2005; TERZIOVSKI; SOHAL, 2000) o del tiempo de fabricación (GRÜTTER et al., 2002). Debemos tener en cuenta que estos beneficios no siempre se consiguen inmediatamente y debe pasar cierto tiempo hasta que se rentabilizan las mejoras incrementales (RAPP; EKLUND, 2002). Sin embargo, hay pocas investigaciones empíricas donde se analicen el impacto a largo plazo de la mejora continua (GRÜTTER et al., 2002). Por lo tanto es necesario realizar investigaciones que evalúen los resultados conseguidos en implantaciones largas.

Nuestra investigación pretende ayudar a cubrir la carencia de estudios de casos longitudinales sobre mejora continua (GRÜTTER et al., 2002). En ella identificaremos las fases que atraviesan el programa de mejora continua en una empresa, los resultados que se obtienen y las diferencias en la implantación de los sistemas de sugerencias y los equipos de mejora. Además, nuestro análisis se realiza en una empresa española del sector de alimentación, de porte mediano, madura, de propiedad familiar y con sistemas de fabricación tradicionales. Es decir, en un contexto muy poco explorado en las publicaciones científicas que se centren en la aplicación de mejora continua en empresas.

\section{Mejora continua}

La mejora continua puede definirse como pequeños cambios incrementales en los procesos productivos o en las prácticas de trabajo que permiten mejorar algún indicador de rendimiento (GRÜTTER et al., 2002), que no necesitan grandes inversiones para realizarse y que cuentan con la implicación de todos los componentes de la empresa (TERZIOVSKI; SOHAL, 2000). Los temas que con más frecuencia son analizados son la mejora de la calidad o costes de fabricación, aunque también son habituales los asuntos de seguridad e higiene en el trabajo (ALBORS; HERVÁS, 2006; BAÑEGIL, 1993; TERZIOVSKI; SOHAL, 2000).

La mejora continua está basada en el ciclo de Deming, compuesto por cuatro fases: estudiar la situación actual, recoger los datos necesarios para proponer las sugerencias de mejora; poner en marcha las propuestas seleccionadas a modo de prueba; comprobar que si la propuesta ensayada está proporcionando los resultados esperados; implantación y estandarización de la propuesta con las modificaciones necesarias (BOND, 1999; BUSHELL, 1992; DEMING, 1993; TERZIOVSKI; SOHAL, 2000).

Existen varias formas de implantar la mejora continua en la empresa. Los mejores resultados se obtienen cuando la mejora se origina en grupos, bien a través de grupos permanentes como en los círculos de calidad (GARCÍA LORENZO; PRADO PRADO, 2001; GRÜTTER et al., 2002; KERRIN; OLIVER, 2002; RAPP; EKLUND, 2002; SILLINCE et al., 1996) o a través de equipos de trabajo multifuncionales o autorregulados que incorporan las actividades de mejora continua entre sus responsabilidades (KERRIN; OLIVER, 2002; RAPP; EKLUND, 2002); o bien, mediante equipos de mejora de duración predeterminada (GARCÍA LORENZO; PRADO PRADO, 2001; GRÜTTER et al., 2002; KERRIN; OLIVER, 2002; RAPP; EKLUND, 2002).

También se pueden implantar sistemas de sugerencias individuales (PRADO, 2001; RAPP; EKLUND, 2002; SCHURING; LUIJTEN, 2001; SILLINCE et al., 1996), aunque sólo obtienen resultados comparables a los grupos si están excepcionalmente bien gestionados (RAPP; EKLUND, 2002).

En nuestra investigación nos centraremos en los programa que añaden una estructura paralela a la que los obreros dedican sólo una parte de su tiempo (de LANGE-ROS; BOER, 2001; SILLINCE et al., 1996; STOHL, 1987). En este sentido, podemos considerar que los programas que aparecieron primero en las empresas fueron los sistemas de sugerencias, seguidos por los círculos de calidad y, más tarde, se introdujeron los equipos de mejora (GARCIA-LORENZO; PRADO, 2003). En un estudio reciente sobre empresas españolas con más de 25 trabajadores, el grado de uso de estos sistemas se decanta claramente hacia los equipos de mejora (presentes en el 74\% de las empresas) y los sistemas de sugerencias (presentes en el 64\% de las empresas). Mientras que los círculos de calidad están dejando de ser habituales (30\% de las empresas) (GARCIA-LORENZO; PRADO, 2003). Creemos que esta situación no es exclusiva de España y el mantenimiento de la popularidad de los sistemas de sugerencias junto con la sustitución progresiva de los círculos de calidad por equipos de mejora también se pueden apreciar en Australia (TERZIOVSKI; SOHAL, 2000) o en Estados Unidos (LAWLER III et al., 2001).

Pasemos a desarrollar con más detalle estos dos tipos más populares de programas para la mejora continua.

Los sistemas de sugerencias individuales proporcionan un procedimiento para recoger, evaluar ideas proporcionadas por los empleados de la empresa (Van DIJK; Van den ENDE, 2002). También permiten formalizar el procedimiento para recompensar a los trabajadores por sus ideas (Van DIJK; Van den ENDE, 2002). Normalmente, los obreros emiten sus sugerencias a través de un buzón de sugerencias (SCHURING; LUIJTEN, 2001), 
rellenando un formulario en papel o formato electrónico. Tradicionalmente, una vez que el obrero ha presentado la idea se desvincula del proceso y la responsabilidad se traslada a un comité que se encarga de seleccionar las ideas premiadas, la cuantía del premio y las personas o grupos que se encargarán de poner en marcha las ideas aprobadas (FRESE et al., 1999; LLOYD, 1999; SCHURING; LUIJTEN, 2001).

Por otra parte, los equipos de mejora comparten bastantes características con los círculos de calidad: están formados por un grupo pequeño de trabajadores que se reúnen periódicamente para identificar, analizar y proponer alternativas de solución de problemas relacionados con su área de trabajo. Ambos grupos solo tienen autonomía para proponer ideas que luego son evaluadas por un comité de mandos que deciden qué ideas deberán ser implantadas. Normalmente la implantación de las ideas corre a cargo de las personas del grupo. Las reuniones se suelen programar en horario de trabajo y no se ofrecen recompensas directas por pertenecer a estos grupos. No obstante, se suelen premiar las ideas en función de su utilidad para la empresa. Estos premios se entregan al grupo para que decida el modo de repartirlos o gastarlos (BARRICK; ALEXANDER, 1987; BUCH; SPANGLER, 1990; GREENBAUM et al., 1988; GRIFFIN, 1988; KERRIN; OLIVER, 2002; LAWLER III, 1991; LI-PING et al., 1988; RAPP; EKLUND, 2002; SILLINCE et al., 1996).

Sin embargo, los equipos de mejora se diferencian en que la participación de los componentes puede no ser voluntaria, sino elegidos por la dirección y suelen pertenecer a diferentes áreas de trabajo o niveles jerárquicos. Esta composición permite propiciar puntos de vista complementarios y abordar problemas que afectan a diferentes áreas. Además, no suelen ser estructuras tan estables como los círculos de calidad en cuanto a duración y miembros que componen el equipo (GARCIALORENZO; PRADO, 2003; LAWLER III et al., 2001; PRADO PRADO, 1998).

\section{Indicadores de resultados}

La medición del rendimiento de la puesta en marcha de programas es una práctica poco habitual (GREENBAUM et al., 1988) pero muy recomendable pues permite a las empresas detectar si los programas están consiguiendo los objetivos perseguidos, diagnosticar las causas que pueden explicar cómo están funcionando y pueden ayudar a identificar posibles alternativas para mejorar la situación (BOND, 1999).

El grado de éxito de la implantación de programas de mejora continua se ha estudiado desde muy diferentes perspectivas. Muchas investigaciones se basan en percepciones subjetivas, bien de los mandos o de los trabajadores, acerca de las ventajas derivadas de la utilización de estos programas. Algunos ejemplos de este tipo son las investigaciones de Sillince et al. (1996) o la de Terziowski y Sohal (2000). Otras, sin embargo, utilizan unos indicadores más objetivos para medir los resultados, como por ejemplo Schroeder et al. (2002).

Además del modo en que se mide el éxito del programa, también hay una gran variación en qué es lo que se mide. Algunos estudios se centran en los resultados financieros (margen de beneficios, retorno de la inversión...), otros ponen el énfasis en los resultados productivos (calidad, nivel de inventario, costes de producción, tiempo de fabricación...), mientras que otros estudios analizan el impacto en indicadores de recursos humanos (absentismo, satisfacción, bajas...) o en los clientes (cuota de mercado, nivel de satisfacción...). Hacer un análisis más detallado de estos aspectos nos alejaría del objetivo de este trabajo y, por otra parte, ha sido tratado en un trabajo reciente (NAIR, 2006), donde se ofrece una bibliografía abundante al respecto.

Por último, existe un conjunto de estudios que analiza el éxito del programa en función de la cantidad de ideas generadas o implantadas y la cantidad de obreros implicados (FRESE et al., 1999; GRIFFIN, 1988; RAPP; EKLUND， 2002; SCHURING; LUIJTEN, 2001; SILLINCE et al., 1996; TERZIOVSKI; SOHAL, 2000; Van DIJK; Van den ENDE, 2002).

Nuestro estudio utilizará unos indicadores similares

Respecto a la evolución que sufre el rendimiento de los programas de mejora, parece haber consenso en que el ciclo de vida de estos programas suele durar entre $24 \mathrm{y}$ 48 meses. En este ciclo podemos identificar tres fases: periodo inicial de introducción, en la que la participación se limita a unas pocas experiencias piloto; difusión de la experiencia en la empresa; decaimiento (algunos autores lo identifican como "efecto luna de miel"), generado por múltiples factores entre los que destacamos la resistencia de los mandos intermedios, el fracaso al implantar algunas de las ideas aprobadas, pocas ideas propuestas porque cada vez es más difícil encontrar temas de mejora o incremento de los costes para mantener en funcionamiento el programa (BARRICK; ALEXANDER, 1987; LAWLER III, 1991; SILLINCE et al., 1996; STOHL; COOMBS, 1988). En algunos casos aparece una cuarta fase, el relanzamiento del programa (RAPP; EKLUND, 2002).

\section{Facilitadores de los programas}

La lista de factores que favorecen el éxito es común para los diferentes programas de mejora continua y la hemos resumido en el Cuadro 1.

Uno de los primeros aspectos es que exista implicación de los trabajadores y de los directivos a través de 
Cuadro 1. Facilitadores del éxito de los programas.

\begin{tabular}{|c|c|}
\hline Facilitador & Autores \\
\hline Implicación de los trabajadores. Motivación intrínseca & $\begin{array}{l}\text { Choi et al. (1997), Fairbank y Williams (2001), Greenbaum et al. (1988), } \\
\text { Sillince et al. (1996) }\end{array}$ \\
\hline Implicación de los directivos & $\begin{array}{l}\text { Greenbaum et al. (1988), Grütter et al. (2002), Lloyd (1999), Prado Prado } \\
\text { (1998), Rapp y Eklund (2002), Schuring y Luijten (2001), Sillince et al. } \\
\text { (1996), Terziovski y Sohal (2000) }\end{array}$ \\
\hline Responsable de mejora continua & Rapp y Eklund (2002) \\
\hline $\begin{array}{l}\text { Formación a los obreros y mandos que participan en el } \\
\text { programa }\end{array}$ & $\begin{array}{l}\text { Bacdayan (2001), Greenbaum et al.(1988), Rapp y Eklund (2002), Sillince } \\
\text { et al. (1996), Terziovski y Sohal (2000), Wood (2003) }\end{array}$ \\
\hline Duración del programa & Barrick y Alexander (1987), Sillince et al. (1996) \\
\hline $\begin{array}{l}\text { Remarcar el interés por obtener cantidad de ideas sen- } \\
\text { cillas }\end{array}$ & Bodek (2002), Choi et al. (1997), Schuring y Luijten (2001), Wood (2003) \\
\hline Divulgar las propuestas aceptadas & Bodek (2002), Fairbank y Williams (2001), Rapp y Eklund (2002) \\
\hline Evaluar rápidamente las propuestas e informar & $\begin{array}{l}\text { Fairbank y Williams (2001), Rapp y Eklund (2002), Schuring y Luijten } \\
\text { (2001), Wood (2003) }\end{array}$ \\
\hline Implantar las propuestas aceptadas rápidamente & Schuring y Luijten (2001), Wood (2003) \\
\hline $\begin{array}{l}\text { Que la persona que hace la propuesta participe en la } \\
\text { implantación }\end{array}$ & Bodek (2002), Schuring (1996) \\
\hline $\begin{array}{l}\text { Recompensar económicamente las propuestas aceptadas } \\
\text { (motivación extrínseca) }\end{array}$ & $\begin{array}{l}\text { Bodek (2002), Fairbank y Williams (2001), Frese et al. (1999), Grütter } \\
\text { et al. (2002), Kerrin y Oliver (2002), Lloyd (1999), Rapp y Eklund (2002), } \\
\text { Schuring y Luijten (2001), Sillince et al. (1996), van Dijk y van den Ende } \\
\text { (2002), Wood (2003) }\end{array}$ \\
\hline $\begin{array}{l}\text { Empezar con experiencias piloto antes de ampliarlo a toda } \\
\text { la empresa }\end{array}$ & Lloyd (1999), Rapp y Eklund (2002) \\
\hline $\begin{array}{l}\text { Definir con claridad los objetivos que se persiguen con el } \\
\text { programa }\end{array}$ & Grütter et al. (2002), Rapp y Eklund (2002), Sillince et al. (1996) \\
\hline $\begin{array}{l}\text { Sistema de selección de ideas transparentes, facilitar la } \\
\text { interacción con los evaluadores }\end{array}$ & Bacdayan (2001), Fairbank y Williams (2001), Wood (2003) \\
\hline Sistema para presentar las sugerencias simple y claras & $\begin{array}{l}\text { Bodek (2002), Fairbank y Williams (2001), Rapp y Eklund (2002), van } \\
\text { Dijk y van den Ende (2002), Wood (2003) }\end{array}$ \\
\hline $\begin{array}{l}\text { Usar indicadores evaluables para identificar el éxito de las } \\
\text { propuestas implantadas }\end{array}$ & Bacdayan (2001), Drach (1994) \\
\hline Formalización de los programas & Grütter et al. (2002), Terziovski y Sohal (2000) \\
\hline Cultura empresarial que estimule la creatividad & $\begin{array}{l}\text { Albors y Hervás (2006), Bacdayan (2001), Fabi y Pons (1995), van Dijk y } \\
\text { van den Ende (2002) }\end{array}$ \\
\hline
\end{tabular}

su participación activa en los comités de supervisión de las sugerencias. Otra forma de demostrar la implicación de la gerencia es mediante la designación de un "responsable de mejora continua" que se encargue de coordinar todas las acciones.

Otro aspecto importante es que la empresa se comprometa proporcionando formación a los participantes en los programas de mejora continua, dándoles a conocer las técnicas que necesitan para poder ir resolviendo problemas más complejos y para mantener el interés de las personas en el programa También es necesario que el programa se mantenga durante suficiente tiempo para que se puedan recoger sus frutos. En caso de sistemas de grupo, que éste se reúna con frecuencia.

También ayuda el remarcar la importancia de recibir cantidad elevada de sugerencias. Y que las propuestas se centren en cambios sencillos, baratos y fáciles de implantar, pues siempre hay tiempo, dinero y personal disponible para poner en marcha pequeños cambios. Y no debemos olvidar que el grado de implantación de las sugerencias realizadas afecta a la motivación para presentar propuestas.

El proceso de evaluación de las ideas debe realizarse en poco tiempo, y se debe informar a los autores de las propuestas. Del mismo modo, el proceso de implantación de las ideas debe ser rápido y en él deberían participar los obreros que proponen las ideas, por eso sería recomendable que las sugerencias de mejora se centraran en el área de trabajo propia.

Los incentivos económicos pueden proporcionar motivación para participar en el programa, sobre todo si, como hacen algunas empresas, se recompensan las buenas ideas aunque no se implanten. La decisión de la cuantía de las recompensas no es una tarea fácil (KERRIN; OLIVER, 
2002), pueden tener una relación con los beneficios que reporta a la empresa, aunque convendría que no fuese directamente proporcional a los ahorros, para evitar que los trabajadores se centren en buscar una idea magnífica que genere un gran ahorro en lugar de presentar gran cantidad de ideas, que es lo que mantiene vivo al sistema (BODEK, 2002; LLOYD, 1999; SCHURING; LUIJTEN, 2001). Algunas empresas consideran que si la sugerencia se ha realizado en horario de trabajo, esas horas ya están pagadas por la empresa y por lo tanto no debe añadirse remuneración adicional. Este es el motivo por el que algunas empresas recompensan sólo las sugerencias individuales y no las emitidas por grupos que se reúnen en horario de trabajo (KERRIN; OLIVER, 2002).

\section{Pregunta de investigación y metodología}

En nuestra investigación pretendemos dar respuesta a los principales interrogantes que se plantean con el análisis de los programas de mejora continua. En concreto, resulta de especial interés conocer las fases que atraviesa el programa de mejora continua en la empresa, los resultados que se obtienen con los sistemas de sugerencias y los equipos de mejora, las diferencias que pueda haber en la implantación de uno y otro programa, así como qué factores de éxito se han activado y cómo.

Para ellos vamos a analizar la evolución de los programas formales de mejora continua de una empresa del sector tradicional (alimentación) a lo largo de 5 años. Se trata de una empresa española, madura, de porte pequeño (aunque cercano a las medianas empresas), de propiedad familiar y cuya filosofía de producción es producción en masa. Todas estas variables han sido destacadas como factores condicionantes del grado de aplicación de sistemas de mejora continua (ALBORS; HERVÁS, 2006; FABI; PONS, 1995; GRÜTTER et al., 2002; SANCHEZ et al., 1999; SILLINCE et al., 1996; TERZIOVSKI; SOHAL, 2000).

Nuestra investigación pretende ayudar a cubrir la carencia de estudios de casos longitudinales sobre mejora continua (GRÜTTER et al., 2002). Además, aunque las preguntas de investigación no son una novedad en el campo académico, consideramos que el contexto donde lo aplicamos sí que lo es. Hasta la fecha, no hemos encontrado publicaciones científicas que se centren en la aplicación de mejora continua en empresas españolas del sector de alimentación y del tamaño de la nuestra. Tampoco son habituales las publicaciones de empresas maduras, de propiedad familiar y con sistemas de fabricación tradicionales (que son la mayoría en España).

Los datos de nuestra investigación han sido recogidos mediante observación participante a lo largo de 18 meses que hemos pasado en la empresa asistiendo a las reuniones de los equipos de mejora.
El estudio de casos conlleva la inevitable limitación de no poder generalizar los resultados obtenidos. Igualmente, cabe la posibilidad de no realizar correctamente el análisis por no haber seleccionado a las fuentes adecuadas, o no haber planteado las preguntas correctas en el momento idóneo. Con el fin de evitar estos errores, nuestra investigación está basada en un protocolo en el que hemos seguido las recomendaciones metodológicas de Yin (1994) y Lange-Ros y Boer (2001). Los datos de la investigación provienen de los archivos de la empresa; de los datos tomados en las reuniones de los equipos y durante la evaluación de las propuestas; de las entrevistas realizadas a 6 directivos de la empresa y de las conversaciones informales con los miembros de los equipos de mejora a lo largo de los meses que hemos pasado en la empresa. Las entrevistas a los mandos de la empresa se realizaron mediante preguntas abiertas enfocadas a obtener la información necesaria sobre las características de la empresa, el funcionamiento de los programas y los resultados obtenidos.

En nuestra investigación, los indicadores de resultados de los programas de mejora continua serán: número de ideas por empleado y año (RAPP y EKLUND, 2002; SCHURING y LUIJTEN, 2001), grado de implantación de ideas: porcentaje de ideas implantadas sobre las recibidas (RAPP y EKLUND, 2002; TERZIOVSKI y SOHAL, 2000; Van DIJK y Van den ENDE, 2002), ahorros generados por las ideas implantadas (en Euros) (Van DIJK y Van den ENDE, 2002).

\section{Estudio del caso}

La empresa FOODSA, está dedicada a la elaboración y comercialización de productos cárnicos procedentes del cerdo y del pavo. Fue fundada hace más de 40 años como una pequeña empresa familiar, en una pequeña planta baja de $125 \mathrm{~m}^{2}$. A mediados de los años $70 \mathrm{se}$ trasladaron a un polígono industrial y diez años después ampliaron las instalaciones hasta $6.000 \mathrm{~m}^{2}$. A partir de 1990, la empresa acomete una fuerte inversión. En el terreno productivo se lleva a cabo una remodelación total de la planta para homologarla a las normas europeas y se amplía hasta el espacio actual (más de $10.000 \mathrm{~m}^{2}$ ). En el ámbito comercial la inversión se materializa con la creación de una red de delegaciones propias.

Su constante compromiso con la calidad hace que en 1997 FOODSA sea certificada según la norma ISO 9002 lo que la convierte en una de las primeras empresas de elaborados cárnicos que obtiene dicha certificación. La calidad de sus productos, la extrema atención de las condiciones higiénicas de sus instalaciones, la infraestructura de su negocio y su espíritu innovador la configuran como una de las empresas líderes. Está situada entre el 10\% de las empresas más grandes y con mejores resultados 
financieros de su sector y ha recibido el reconocimiento de diversas instituciones públicas.

Actualmente, la empresa está regentada por la tercera generación de la familia. Podemos considerarla como una organización "tradicional" con cultura burocrática, utilizando el uso de reglas, políticas, autoridad jerárquica, documentación escrita y sistemas de recompensas para influir sobre el comportamiento de sus empleados y valorar su rendimiento. Sin embargo, desde hace unos años se está produciendo un giro en el que se está intentando un cambio en la cultura empresarial orientada a fomentar la implicación de los obreros en la toma de decisiones relacionadas con su tarea o ámbito de trabajo y la mejora continua de la empresa, propiciando un entorno más dinámico y flexible.

La fabricación de los productos se realiza contra inventario. Para ello se tienen en cuenta los datos históricos, las previsiones de ventas para el último mes y la tendencia de la venta de los productos. Al tratarse de productos perecederos, el stock de seguridad se ha de controlar para que no supere los doce días. El stock de seguridad se revisa cada semana en función de lo fabricado y se actualiza periódicamente.

En el proceso de producción se pueden distinguir las siguientes secciones: recepción de mercancías, procesos de previos fabricación (descongelación, preparación, picado, inyección, amasado), embutición, tratamiento térmico, procesos específicos (desmoldeado y loncheado), envasado, almacenaje y expediciones.

La distribución en planta tiene forma de $\mathrm{U}$. El producto entra por la misma zona por donde se expide. Esta forma de ubicar las zonas de trabajo permite, mayor flexibilidad para adaptarse a cambios de la demanda, aumentando o disminuyendo el número de trabajadores dentro de una línea y el número de tareas de cada uno de ellos.

El número de referencias se aproxima a 200, agrupadas en tres familias: productos cocidos, productos curados y productos frescos.

\subsection{El programa de mejora continua de la empresa}

En enero de 2000 se inició el programa de mejora continua en FOODSA. La iniciativa partió de la gerencia de la empresa y este programa inicial sentó las bases de lo que es el actual programa de mejora continua.

Las propuestas se deben centrar en mejoras que no toquen temas sindicales (salarios, categorías, tiempo de trabajo, convenio colectivos...) o que dependan directamente de la gerencia de la empresa (relaciones públicas, publicidad, derechos y obligaciones de la empresa...).Tampoco se pueden proponer estudios o procedimientos ya en curso de desarrollo o análisis en las áreas de actividad de la empresa ni sugerencias que impliquen el cambio de las fuentes de suministro.
En los orígenes del programa, las sugerencias se depositaban en un buzón colocado a la vista de todos y se recogían semanalmente. Pero este método no conseguía fomentar la participación de los empleados, por lo que se pasó a entregar las propuestas en el departamento de recursos humanos.

Actualmente, las propuestas de mejora pueden provenir tanto de empleados individuales, como de grupos de empleados. Quedan excluidos del sistema de sugerencias o equipos de mejora los directivos y técnicos de la empresa.

\subsection{La experiencia del sistema de sugerencias}

El primer año de existencia del programa de sugerencias no se realizó un seguimiento de las propuestas, que fueron muy escasas. A partir del año 2001 se dispone de algunos datos. Cuando se inició nuestra investigación en 2004 se hizo un seguimiento más detallado, registrando de manera sistemática las propuestas de ideas y la viabilidad de las mismas.

Para presentar una idea o sugerencia, se debía cumplimentar un impreso normalizado. La presentación de las sugerencias podía variar ligeramente de formato con tal de que la idea se quede perfectamente definida y que quedasen reflejados los requisitos básicos exigibles para la presentación de la misma: el nombre del autor o autores, la fecha de entrega de la propuesta, el problema o mejora detectados, el tipo de solución propuesta (mejora de calidad, mejora del proceso productivo, mejora del proceso administrativo, logística), la solución propuesta, el croquis de la solución, si fuese necesario, y firma de los autores.

Una vez completado el impreso, se debía remitir al departamento de recursos humanos, para que éste, y en función de la finalidad de la idea, lo remitiera al departamento correspondiente para que la estudiase. El departamento en cuestión evaluaba en el plazo más breve posible su viabilidad, efectividad, coste, mejoras introducidas, ahorro que supone, inconvenientes generados...y emitía un informe sobre la propuesta. El informe de evaluación se remitía al departamento de recursos humanos que se encargaba de transmitirlo a la persona o grupo de personas que habían formulado la propuesta. En muchas ocasiones, las ideas quedaban estancadas en los departamentos sin que se produjera la evaluación o ésta no era del todo rigurosa. Por este motivo se cambió el procedimiento en 2004 y las propuestas pasaron a evaluarse por una persona encargada de dinamizar el sistema de mejora continua (para el cálculo de los ahorros, se siguieron las directrices descritas en el Anexo).

$\mathrm{Si}$ se presentaban propuestas que no eran viables en el momento de su valoración, pero pudieran serlo en un plazo de un año a partir de su presentación, la propuesta quedaba en espera de que se produjeran las condiciones 
oportunas para su puesta en marcha. Las ideas implantadas se van publicando en la hoja informativa mensual de la empresa.

Las ideas aprobadas reciben una gratificación económica que está en función del ahorro calculado en el informe de la propuesta. Las ideas que ahorran menos de $3.000 € /$ año son premiadas con el $6 \%$ del ahorro, con un límite de $150 €$. Si el ahorro está entre 3.000 y $30.000 € /$ año perciben el $5 \%$, es decir, entre $150 € \mathrm{y}$ $1.200 €$ de gratificación. Los ahorros entre 30.000 y $60.000 € /$ año reciben el $4 \%$ (1.200 y $2.100 €)$. Los ahorros superiores a $60.000 € /$ año reciben el $3.5 \%$, con un límite de $6.000 €$ de premio.

Además, en el caso de que una idea no produzca ahorro porque su implantación requiera de una inversión mayor que los beneficios generados, pero la dirección de la empresa considere oportuno implantarla por alguna razón (seguridad de las personas, orden, limpieza, etc.) se entrega a los autores de la idea una gratificación simbólica de $60 €$.

Los autores de ideas que se consideran no aptas (por razones técnicas o económicas) entran en un concurso de un lote de productos. La finalidad de este concurso es agradecer el esfuerzo y la contribución de las personas de la empresa a la mejora continua.

La propiedad intelectual de las ideas implantadas y gratificadas pasa a ser de la empresa FOODSA.

En la Tabla 1 mostramos los principales indicadores de resultados del sistema. En ella podemos apreciar que el número de ideas presentadas es muy variable. Después de 24 meses el número de propuestas, así como su grado de implantación, decae. Durante el año 2003 se hizo un esfuerzo por parte de los mandos de producción para potenciar el sistema de sugerencias. El resultado fue un aumento espectacular de propuestas presentadas, aunque muchas de ellas no fueron aceptadas. Durante al año 2004 se incrementa el compromiso de la dirección con el programa de mejora continua y se contrata un técnico a tiempo completo para que actúe como "responsable de mejora continua", porque la acumulación de propuestas sin evaluar estaba a punto de causar la muerte del programa de sugerencias. Además de sistematizar el cálculo de los ahorros y la evaluación de las ideas, el responsable de mejora continua se encargó de lanzar una experiencia piloto con dos equipos de mejora que se mantuvo en paralelo con el sistema de sugerencias y que detallaremos en la sección siguiente.

Consideramos destacable el hecho de que en el año 2005 se volviera a unos niveles de presentación de sugerencias del año 2001, sin embargo, el índice de aceptación de propuestas es muy elevado (78\%) lo que permite mantener un ahorro anual similar al del año anterior. De los tres primeros años no se disponen de datos del cálculo de los ahorros por haber sido realizados por personas diferentes, pertenecientes a varios departamentos, que no siempre usaron los mismos criterios. Además no se guardaron copias de los informes de evaluación realizados.

Las áreas que generaron más propuestas fueron: embutición $(32 \%)$, loncheado $(20 \%)$, productos cocidos $(15 \%)$ y empaquetado (10\%). El resto de secciones productivas o departamentos de la empresa contribuye en menos del $5 \%$.

Las secciones de embutición y la de productos cocidos, son secciones poco automatizadas, en las que trabajan bastantes obreros. En cambio, la sección de loncheado dispone de líneas de trabajo muy automatizadas. Sin embargo, la automatización de la sección de loncheado es relativamente nueva (año 2000). Otro factor favorecedor de la cantidad de propuestas presentadas en loncheado es la insistencia por parte de la dirección de producción para fomentar las propuestas de mejora en esta sección.

En cuanto a la contribución al ahorro durante el año 2004, las secciones que más han contribuido son: mantenimiento e instalaciones con un $43 \%$, seguido por loncheado con un $18 \%$. La explicación más probable es que las secciones de mantenimiento e instalaciones son las que tienen acceso directo a más información, lo que les permite comprobar la viabilidad de sus ideas. Vuelve a destacar en este apartado la sección de loncheado, posiblemente porque al ser líneas de reciente creación los obreros son capaces de realizar mayor número de aportaciones.

Hasta el año 2004, en el sistema de sugerencias no se lleva un seguimiento del ahorro conseguido por el sistema.

Tabla 1. Resultado del sistema de sugerencias.

\begin{tabular}{|c|c|c|c|c|c|}
\hline & 2001 & 2002 & 2003 & 2004* & $2005 * *$ \\
\hline $\mathrm{N}^{\mathrm{o}}$ obreros $* * *$ & 193 & 170 & 172 & 165 & 179 \\
\hline $\mathrm{N}^{\mathrm{o}}$ propuestas & 105 & 76 & 325 & 148 & 94 \\
\hline Propuestas/empleado/año & 0,54 & 0,45 & 1,89 & 0,90 & 0,52 \\
\hline$\%$ propuestas implantadas & $57 \%$ & $49 \%$ & $39 \%$ & $59 \%$ & $77 \%$ \\
\hline Ahorro (€/año) & n.d & n.d & n.d & $51.200 €$ & $51.600 €$ \\
\hline
\end{tabular}

* Durante este año el sistema de sugerencias coexistió con la experiencia piloto de equipos de mejora (en el número de obreros hemos restado las 10 personas participantes en la experiencia piloto); ** Extrapolando los datos disponibles (meses de enero a mayo); *** Aproximadamente el $50 \%$ de los obreros tienen contrato fijo. 
El cálculo y la posterior divulgación de los ahorros producidos por el sistema de sugerencias en el año 2004 han generado en los obreros un cambio de actitud hacia el programa de sugerencias. Los trabajadores perciben que las ideas que aportan no caen en el olvido. También se percibe que los trabajadores se sienten implicados en los cambios y en las decisiones que afectan a la empresa y se sienten satisfechos por ello.

Han aparecido problemas a la hora de cuantificar el ahorro de las propuestas relacionadas con limpieza, orden, ergonomía, etc. Para resolverlos se propone dar un premio simbólico (entre 12 y $30 €$ ) para estas propuestas. La cuantía será determinada por el departamento de recursos humanos en función de la creatividad de la propuesta.

A pesar de que los directivos consideran que el sistema de sugerencias está dando buenos resultados, se producen algunos efectos no deseados. Por ejemplo, a veces se presenta la reparación de una avería como una propuesta del sistema de sugerencias. También se han detectado algunas propuestas carentes de esfuerzo o creatividad, cuyo único objetivo es permitir al obrero participar en el concurso de fin de año.

\subsection{Experiencia piloto con equipos de mejora}

A mediados del año 2004 se crearon dos equipos de mejora. Los aspectos sobre los que debían trabajar eran la mejora de la calidad del producto, reducción del porcentaje de mermas, ahorro de tiempos de cambio de partida y limpieza, ahorro de tiempos de ciclo de máquina, estandarización de las operaciones, aumento de productividad o ergonomía.

Estos equipos estaban formados por coordinadores de pequeños grupos de obreros, es decir, personal cualificado, con nivel de estudios de graduado escolar o FPII. Los componentes de los equipos recibieron 20 horas de formación específica acerca de las características de los grupos de mejora y sus objetivos, y las herramientas para la mejora continua.

Cada uno de los dos grupos estaban compuestos por cinco personas de distintas áreas de la empresa (recepción de mercancías, desmoldeo, empaquetado, loncheado, productos cocidos, instalaciones, mantenimiento...). Esto les permitía compartir conocimientos y puntos de vista complementarios, comprender mejor el trabajo que realizan en otras secciones y aprender de las experiencias de los demás.

A las reuniones asistía también el Director general de la empresa, en la medida en que su agenda se lo permitía, y el responsable de mejora continua, que actuaba como coordinador de las reuniones (convocaba las reuniones, moderaba las reuniones, anotaba por escrito los acuerdos...). El responsable de mejora continua participaba en el análisis de la viabilidad de las propuestas $\mathrm{y}$, junto con los directivos de la empresa, seleccionaba cuáles se implantarían. Las reuniones se realizaban quincenalmente, dentro del horario laboral y su duración era, normalmente, de 30 minutos.

En la reunión inicial se establecieron un calendario y el horario de las reuniones. Las siguientes reuniones se dedicaron a completar los pasos necesarios para rellenar las propuestas: identificación de las áreas que precisan mejoras; análisis de los procesos; generación de distintas alternativas de solución; análisis de los costes, ventajas e inconvenientes de las alternativas (en la medida de lo posible se cuantificaba cada uno de estos aspectos para ayudar a la toma de decisiones); documentar cómo pueden llevarse a la práctica las mejoras; consultar con la viabilidad con los responsables de las secciones o departamentos implicados y resumir toda esta información en un formulario de propuesta.

Si la propuesta era aceptada por la dirección, se implantaba y se comprobaba durante un periodo prudencial si era tan eficaz como se esperaba, actuando en consecuencia. Al finalizar el año, ambos equipos hicieron una presentación de los avances conseguidos en una reunión a la que acudieron los directivos de la empresa, así como una delegación del consejo de administración.

El área de estudio seleccionada para la experiencia piloto fue la sección de loncheado, que se eligió por diversos motivos. Por un lado se trataba de una de las secciones más activas en el sistema de sugerencias. Por otro, los productos loncheados representan un elevado porcentaje de las ventas de la empresa. Además, estaba previsto realizar reformas en otras secciones y ésta era una de las secciones que iba a permanecer inalterable en la redistribución de la empresa.

Las salas donde se realiza el loncheado son un lugar altamente higienizado y estanco, en el que se siguen unas normas de seguridad severas para la manipulación de alimentos. Con ello se consigue reducir los riesgos de contaminación del producto durante las tareas de corte y envasado así como prolongar su conservación sin necesidad de recurrir a agentes químicos (conservantes). La empresa dispone de un total de cuatro salas donde se procesan anualmente cerca de 3.000 toneladas de producto loncheado en unas condiciones higiénicas extremas. En cada sala hay una línea de fabricación con 4 obreros trabajando a tres turnos. En total, en la sección trabajan 48 operarios.

Una parte de los ahorros anuales de cada sugerencia implantada se destina a gratificar a los miembros del equipo de mejora que la ha propuesto. Las propuestas que generan ahorros menores que $6.000 € /$ año reciben un "regalo de la empresa" (a determinar en cada ocasión). Si la propuesta ahorra entre 6.000 y $15.000 € /$ año, se entrega al grupo un $3 \%$ de esa cantidad como premio. $\mathrm{Si}$ 
los ahorros superan los $15.000 € /$ año se premia con un $6 \%$ (con un límite de $6.000 €$ ).

Cada grupo convocó 9 reuniones, con una asistencia global del $80 \%$ de componentes. El resultado fueron 28 propuestas presentadas, de las cuales se implantaron 23. Tras un periodo de prueba, se confirmó que el conjunto de propuestas implantadas supondría un ahorro de más de $105.000 € /$ año (Tabla 2). A esto hay que añadir que se generó alguna propuesta que requería de una gran inversión y que se pospuso por falta de recursos, por lo que no se ha incluido como propuesta implantada. Sólo una propuesta generó más de $15.000 € /$ año de ahorro y 17 de las ideas implantadas generaron menos de $6.000 € /$ año de ahorro cada una (en conjunto el 39\% de los ahorros). La cantidad total repartida en premios, sin contar los "regalos de empresa", fue de $2.500 €$ (un $2,3 \%$ de los ahorros).

A pesar de la satisfacción por los resultados de los equipos, en estos meses de experiencia piloto, se ha podido observar algunas deficiencias en el sistema. Por ejemplo, existen diferencias entre la gratificación acordada para el sistema de sugerencias y el de los equipos de mejora. A los miembros de los equipos les cuesta entender que el mantenimiento del programa de equipos de mejora obliga a la empresa a realizar inversiones que no son necesarias para el sistema de sugerencias. Por lo tanto el programa de equipos intenta incentivar la generación propuestas muy rentables (más de $15.000 €$ /año de ahorro), en detrimento de las de ahorros discretos (menos de $6.000 € /$ año). Este problema es similar al comentado por Schuring y Luijten (2001). Adicionalmente, el hecho de que el premio para las propuestas con ahorros menores de $6.000 € /$ año no estuviese establecido, generó algunos problemas.

También han surgido problemas con el pago de las recompensas. Estas se entregan al finalizar la experiencia piloto, no como en el programa de sugerencias que se entregan una vez que ha sido aprobada la idea. Por lo tanto, el premio por el esfuerzo se cobra 8 meses después de haber empezado a proponer e implantar ideas y pierde gran parte de su capacidad motivadora.

Otro problema es que los participantes en los equipos se han sentido coaccionados a participar por temor a las posibles represalias si no aceptaban la invitación a formar parte de los equipos. A pesar de que estos temores eran totalmente infundados, se generó cierta resistencia que dificultó la cohesión del equipo.
La mayoría de los problemas y oportunidades de mejora planteados al principio de la experiencia piloto fueron identificados por la dirección general de la empresa. Los componentes del grupo aún no han adquirido del todo la capacidad para detectar con autonomía los problemas u oportunidades.

El director general asistió a las primeras reuniones y los obreros percibieron el interés de la alta dirección. Esto produjo motivación. Sin embargo, a medida que pasó el tiempo, los directivos dejan de acudir y los operarios observan este hecho, lo que les lleva a pensar que lo que dicen carece de importancia y que no será tomado en cuenta, con la desmotivación consiguiente.

Sin embargo, la presencia del director general en las reuniones no siempre ha sido positiva. En las reuniones en las que estaba presente se observaba un comportamiento "conservador" de los obreros, con menos fluidez de ideas innovadoras. No obstante, este problema se fue amortiguando a medida que los obreros se acostumbraron a la presencia del directivo.

También aparecen una falta de comunicación y coordinación entre departamentos, especialmente con el departamento de Investigación y Desarrollo (I + D). En algunos casos se realizaban pruebas de productos duplicadas, perdiendo tiempo y esfuerzo y no se aprovechó información que podía haber sido muy útil tanto para los equipos de mejora como para I + D.

Además, como no se informó demasiado sobre el lanzamiento de la experiencia piloto, algunos mandos intermedios llegaron a pensar que se estaba interfiriendo en sus funciones. Además consideraban que cualquier actividad extra relacionada con estos equipos, por ejemplo, buscar información en los archivos, realizar algún tipo de prueba de producto o colaborar en la implantación de una propuesta, suponía una traba para realizar su "verdadero" trabajo.

Otro problema fue que en algunos departamentos no se reconocía la autoridad del responsable de mejora continua o consideraban que su trabajo era transitorio, a pesar de contar con el respaldo de la dirección general de la empresa, de la que depende directamente. Esto produjo retrasos en la entrega de información disponible en los archivos, obligando a obtenerla por otros cauces.

Por último, se pretendió abarcar demasiados temas al principio. Se amontonaban las ideas sin llegar a formalizar las propuestas, especialmente las más sencillas y que por lo tanto, hubiesen producido ahorros pequeños sin gratificación económica. Los equipos sentían que no

Tabla 2. Resultado de los equipos de mejora (6 meses, año 2004).

\begin{tabular}{ccccc}
\hline $\mathbf{N}^{\mathbf{0}}$ participantes & $\mathbf{N}^{\mathbf{0}}$ propuestas & $\begin{array}{c}\text { Propuestas por } \\
\text { empleado/año }\end{array}$ & \% propuestas implantadas & $\begin{array}{c}\text { Ahorro (€/año) de las } \\
\text { propuestas implantadas }\end{array}$ \\
\hline 10 & 28 & 2,8 & $82 \%$ & $106.581 €$ \\
\hline
\end{tabular}


se avanzaba ni se ejecutaban las ideas y se generó cierta desmotivación.

\section{Discusión}

Respecto a la primera de nuestra preguntas de investigación, hemos podido comprobar que el programa de sugerencias ha atravesado las etapas propuestas por la literatura científica: tras unos inicios titubeantes, se consiguió una participación aceptable en cuanto a número de propuestas presentadas (bastantes de las cuales se pusieron en marcha) con niveles similares a los mostrados por Frese et al. (1999) y Schuring y Luijten (2001) y bastante menores que los de Rapp y Eklund (2002). En el segundo año de existencia aparece el "efecto luna de miel" (LAWLER III, 1991), pero en lugar de desaparecer el programa, se entra en una fase de relanzamiento (RAPP; EKLUND, 2002) que hay que ir animando cada año: con arengas de los directivos; recogiendo la valoración económica de las propuestas y divulgándola entre los obreros; creando nuevos sistemas como los equipos de mejora y, probablemente, extendiendo la formación sobre herramientas de mejora continua (BACDAYAN, 2001; GREENBAUM et al., 1988; RAPP; EKLUND,
2002; SILLINCE et al., 1996; TERZIOVSKI; SOHAL, 2000; WOOD, 2003). En este sentido, los datos extrapolados del 2005 parecen indicar que se acerca el peligro de un nuevo estancamiento por falta de propuestas (BODEK, 2002; LLOYD, 1999; SCHURING; LUIJTEN, 2001). Aunque las que se presentan son muy buenas, se implantan casi todas y logran unos ahorros similares a los del año anterior.

En el Cuadro 2 resumimos las principales diferencias de funcionamiento de ambos programas. Resaltamos que los equipos de mejora hacían una presentación resumen de los logros de la experiencia piloto ante la gerencia de la empresa e interactuaban con los directivos en las reuniones quincenales. Por lo tanto, formar parte de los equipos de mejora, además de la satisfacción personal que supone el reconocimiento de los altos mandos de la empresa y de los demás empleados, podía servir de plataforma de promoción de los obreros. Esto aumentaba la motivación interna para participar y hacerlo bien, que es uno de los principales requisitos para el éxito del sistema (CHOI et al., 1997; FAIRBANK; WILLIAMS, 2001; GREENBAUM et al., 1988; SILLINCE et al., 1996).

El sistema de recompensas en los equipos fue causa de problemas. Por un lado, es más difícil de conseguir

Cuadro 2. Comparación de los dos sistemas.

\begin{tabular}{|c|c|c|}
\hline Variable & Sistema de sugerencias & Equipos de mejora \\
\hline Estado de formalización & Consolidado. 5 años en marcha. & Experiencia piloto. \\
\hline Participantes & $\begin{array}{l}\text { Todos los obreros de la empresa. Voluntarios. } \\
\text { Individualmente o como grupo. }\end{array}$ & $\begin{array}{l}\text { "Voluntario" por invitación. Sólo dos grupos. } 10 \text { par- } \\
\text { ticipantes. Empleados selectos. }\end{array}$ \\
\hline Formación ofrecida & Ninguna & 20 horas sobre solución de problemas en grupo \\
\hline Divulgación & Programa muy conocido & $\begin{array}{l}\text { Apenas es conocido el programa por las personas no } \\
\text { participantes }\end{array}$ \\
\hline Recompensas & $\begin{array}{l}\text { Económicas. Proporcionales a los ahorros } \\
\text { generados. Se pagan tras la aceptación de la pro- } \\
\text { puesta. Se priman las ideas sencillas y fáciles de } \\
\text { implantar. Premios de consolación simbólicos. }\end{array}$ & $\begin{array}{l}\text { Económicas a partir de ahorros superiores a los } \\
6.000 € \text { Se pagan al finalizar la experiencia piloto. } \\
\text { Se priman las ideas que generan grandes ahorros. }\end{array}$ \\
\hline Implicación de la dirección & No visible & $\begin{array}{l}\text { Coordinador de mejora continua. Asistencia de los } \\
\text { directivos a las reuniones para ayudar a identificar } \\
\text { temas y valorar las propuestas }\end{array}$ \\
\hline Presentación de propuestas & $\begin{array}{l}\text { Formulario sencillo. Presentado en departamento } \\
\text { de recursos humanos }\end{array}$ & $\begin{array}{l}\text { Formulario sencillo (más completo que el de suge- } \\
\text { rencias) rellenado a partir de los datos de las actas de } \\
\text { las reuniones. La propuesta la presenta el responsa- } \\
\text { ble de mejora continua a la dirección de la empresa }\end{array}$ \\
\hline $\begin{array}{l}\text { Valoración económica de los } \\
\text { ahorros (cuantificación de } \\
\text { propuestas) }\end{array}$ & $\begin{array}{l}\text { Comité externo. Directivos de diferentes depar- } \\
\text { tamentos. Criterios no uniformes. Tardan mucho } \\
\text { en valorar }\end{array}$ & $\begin{array}{l}\text { Coordinador del sistema de mejora continua. Valora- } \\
\text { ción de las propuestas en un plazo breve (una o dos } \\
\text { semanas). Criterios públicos. }\end{array}$ \\
\hline $\begin{array}{l}\text { Aceptación/rechazo de las } \\
\text { propuestas }\end{array}$ & Dirección de la empresa & Dirección de la empresa \\
\hline Implantación de las ideas & $\begin{array}{l}\text { Inmediata tras la aprobación. A veces participan } \\
\text { los autores de la propuesta }\end{array}$ & $\begin{array}{l}\text { Inmediata tras la aprobación. Casi siempre partici- } \\
\text { pan los miembros del equipo }\end{array}$ \\
\hline $\begin{array}{l}\text { Impresiones de los directivos } \\
\text { sobre el éxito del programa }\end{array}$ & $\begin{array}{l}\text { Satisfactorios. Apenas cuesta dinero y genera } \\
\text { ahorros. Es necesario innovar el sistema para } \\
\text { fomentar la participación }\end{array}$ & $\begin{array}{l}\text { Muy satisfactorios. Los ahorros son muy superiores } \\
\text { a la inversión para mantener el programa. Se piensa } \\
\text { cómo extenderlo y compatibilizarlo con el sistema } \\
\text { de sugerencias. }\end{array}$ \\
\hline
\end{tabular}


pues la recompensa no está en función de los ahorros estimados, sino de la comprobación de los ahorros tras la verificación después de un periodo de prueba. Además, los porcentajes entregados para las propuestas con ahorros pequeños (las más abundantes) son menores que en el sistema de sugerencias y, por último, se tarda bastante en percibir la recompensa económica. Estos problemas son similares a los comentados por Kerrin y Oliver (2002).

Otra dificultad fue que, a pesar de que se hizo mención de los equipos de mejora en el boletín trimestral de noticias internas, los obreros y mandos intermedios de la fábrica no se enteraron de la creación de los equipos ni de sus atribuciones. No obstante, a medida que avanzaron los meses, se fue difundiendo la información, bien por boca de sus compañeros o por el responsable de mejora continua.

Por último, al comparar la aplicación real (Cuadro 2) con las recomendaciones teóricas (Cuadro 1), podemos comprobar que la mayoría de las recomendaciones contenidas en las publicaciones científicas han sido adoptadas por la empresa, salvo los problemas comentados con la tardanza en evaluar las propuestas en el sistema de sugerencias y los de las recompensas y la publicidad del sistema en los equipos de mejora.

Estos factores de éxito se han ido incorporando de forma progresiva. Algunos de ellos estaban presentes en el esquema inicial del sistema de sugerencias. Otros se han ido añadiendo para paliar las disfunciones del sistema y otros formarán parte de la propuesta del nuevo sistema que se pretende implantar después de la experiencia piloto con los equipos de mejora. No en balde, la empresa ha aprendido que el éxito de los programas de mejora continua sólo se mantiene en el tiempo si se aplican los esfuerzos necesarios para revitalizarlos y mantener el interés de los empleados.

Así, por ejemplo, se presentará una precampaña para avisar de la creación de los equipos de mejora y el procedimiento para inscribirse. En el nuevo sistema, los obreros, podrán escoger dos caminos paralelos. Participar en un programa sencillo en el que se emite la sugerencia y se queda libre de obligaciones (sistema de sugerencias), o participar en un programa más elaborado, en grupos, donde después de emitir la sugerencia, tiene la posibilidad de aprovechar diversos recursos que le ofrece la empresa y participar en la implantación de las ideas (equipos de mejora). Estos equipos podrán ser de libre asociación o propuestos por la dirección.

Las recompensas serán un porcentaje del ahorro conseguido el primer año después de la implantación de la idea. Este porcentaje será fijado por la dirección general de la empresa. También se ofrecerán premios simbólicos para las ideas que generen poco ahorro con el fin de mantener la motivación.
Al analizar comparativamente los resultados de ambos sistemas durante el periodo que han coexistido, las cifras pueden resultar engañosas. La cantidad de propuestas de los equipos de mejora, el grado de aceptación y los ahorros generados son llamativamente superiores a los resultados de los sistemas de sugerencias, sobre todo, si pensamos que en los equipos de mejora han participado sólo 10 obreros y han estado funcionando sólo 6 meses.

Sin embargo, debemos tener en cuenta algunos factores que diferencian ambas implantaciones. Por un lado, el mantenimiento del sistema de sugerencias apenas genera gastos a la empresa, salvo unas pocas horas de los directivos de los departamentos evaluando las propuestas. Sin embargo, el mantenimiento de los equipos de mejora obliga a realizar unos gastos fijos: el sueldo del responsable de mejora continua, la parte proporcional del salario de directivos y operarios correspondiente a las horas invertidas en las reuniones y los gastos de formación (GRIFFIN, 1988).

Además, los excelentes resultados de los equipos de mejora se han podido conseguir gracias a la participación y guía de los directivos y técnicos que colaboraban en la identificación de propuestas o la generación de alternativas. Y, por si no fuese suficiente, los equipos de mejora se formaron con los obreros que mejores evaluaciones de desempeño habían recibido el año anterior y que actuaban como coordinadores del trabajo de sus compañeros. Es decir, los obreros más preparados y con más experiencia.

Con esto no queremos restar mérito a los resultados conseguidos por los equipos, sino remarcar que extender el programa de equipos de mejora a más trabajadores no generaría resultados proporcionales a los actuales, porque los costes de mantenimiento se dispararían, los directivos no podrían implicarse como se han implicado en la experiencia piloto, los obreros no estaría tan capacitados o motivados como los 10 seleccionados en la experiencia piloto, el programa ya no sería una novedad (efecto luna de miel) y en algún momento se agotarían los problemas abordables.

En definitiva, aunque es cierta la afirmación de que los equipos de mejora dan mejores resultados que los sistemas de sugerencias (RAPP; EKLUND, 2002), probablemente no sea recomendable empezar directamente con equipos de mejora, sino que esta sea la evolución natural para mantener vivo un sistema de mejora continua que se inicia con sistemas de sugerencias (menos costosos de mantener y más fáciles de implantar) (LAWLER III, 1991).

En cualquier caso, las conclusiones de esta investigación no son extrapolables fuera del contexto particular estudiado, empresa familiar española mediana con sistemas de fabricación en masa, perteneciente a sector tradicional. 
Otra limitación ha sido la falta de datos históricos del ahorro proporcionado por el sistema de sugerencias antes de nuestra intervención. Tal como comentan Greenbaum et al. (1988) y Drach (1994) no es habitual encontrar en las empresas el grado de disciplina y sistematización suficiente para recoger y mantener estos datos.

Por último, nos falta perspectiva para valorar la evolución los dos sistemas en el futuro y las preferencias de los obreros. Nos gustaría continuar la investigación para descubrir si uno de los sistemas se impondrá sobre el otro y si el sistema de equipos de mejora puede superar su fase de luna de miel y continuar con resultados positivos si se amplía la propuesta a toda la empresa.

\section{Conclusiones}

A lo largo de nuestra investigación hemos podido comprobar que no existe una fórmula mágica para el correcto funcionamiento del sistema de mejora continua, sino que hay que tratar de mejorar continuamente el sistema existente, puliendo fallos y tratando de aportar siempre algo nuevo que relance cada cierto tiempo el sistema. En este sentido, nuestros datos ponen de manifiesto una experiencia real de cómo se ha ido transformando un esquema de mejora continua, partiendo de unos comienzos nada exitosos, atravesando diferentes fases y aportando resultados para la empresa.

A partir de un programa de sugerencias, relativamente fácil y económico de implantar, se inició en la empresa el germen de una cultura de implicación del personal en la mejora continua. No obstante, se necesita un empuje y una revitalización constante por parte de la dirección para mantener los buenos resultados iniciales. El apoyo inequívoco de la alta dirección en este contexto es crucial, demostrado en nuestro caso con la contratación del responsable de mejora continua y con el esfuerzo por realizar un seguimiento adecuado del programa y por generalizar la difusión de los resultados conseguidos.
La evolución natural del anterior sistema ha sido hacia un programa de equipos de mejora. La infraestructura de este programa es más costosa de mantener, por lo que los objetivos se orientan más a propuestas que generasen un ahorro mayor. Por otra parte, su mayor complejidad también vino acompañada de dificultades en su implantación, pese a que la práctica de la totalidad de las recomendaciones que aparecen en la teoría fueron adoptadas por la empresa. Destaca el hecho de que la alta dirección, de nuevo, muestra su respaldo a este programa, introduciendo las mejoras necesarias en cuanto a difusión de la información y organización de los equipos.

Esta investigación nos ha permitido remarcar la importancia de la mejora continua en la empresa tanto desde el punto de vista económico como del desarrollo de los trabajadores. Hemos podido comprobar el papel tan importante que desempeñan los trabajadores en este tipo de programas y cómo se puede ir avanzando paulatinamente para llegar a conseguir que todo el personal de la empresa (mandos y obreros) vaya percibiendo la necesidad de la mejora continua como herramienta fundamental de competitividad. Es decir, de inculcar, de alguna forma, la cultura de empresa necesaria para llevarla a cabo, dar fluidez y fomentar el correcto funcionamiento de los sistemas de sugerencia y los equipos de mejora.

La contribución de este trabajo a la comunidad académica radica en la utilidad de los estudios longitudinales como herramienta de investigación. Sólo a partir de un seguimiento continuado en una empresa se pueden detectar no sólo la evolución de diferentes programas, sino la problemática detallada ante la que se enfrentan y los modos de resolverla. En cuanto al mundo empresarial, las organizaciones interesadas en aplicar programas de mejora continua tienen ante ellas un interesante caso práctico que les ilustrará cómo todos los comienzos son tímidos, complejos y difíciles, y especialmente cómo los objetivos a largo plazo pasan por introducir en la cultura de la empresa los elementos básicos para involucrar a todos los miembros en la mejora continua. 


\section{Estudo longitudinal dos resultados da melhora continua em uma empresa industrial}

\section{Resumo}

As empresas tem utilizado diversas ferramentas que permitem que os operarios comtribuam ao processo de melhora continua. Entre as ferramentas mas usadas podemos destacar os sistemas de sugestoes tanto individual como em grupo. Nesta comunicação faremos um repaso das principais caracteristicas de ambos sistemas e os modos habituais de implantação. Nossa comunicação pretende responder a estas perguntas de pesquisa. Que resultados derivam da implantaçao de sistemas de sugestoes individuais ou em grupo? Qual dos dois sistemas beneficia mais a empresa? Que problemas surgem durante o funcionamento destes programas?

Para isso analisaremos os dados de um caso de empresa industrial, onde tiramos os dados historicos de 5 anos de aplicação de um programa melhora continua. Ambos programas demonstraram ser aproveitados para a empresa, ainda que as possibilidades dos sistemas de grupo se mostram significativamente maiores.

Palavras-chave: Melhora continua. Sistemas de sugestões. Equipes de melhora. Resultados. Redução de custos.

\section{Referencias bibliográficas}

ALBORS, J.; HERVÁS, J. L. CI practice in Spain: its role as a strategic tool for the firm. Empirical evidence from the CINet survey analysis. International Journal of Technology Management, v. 35, n. 5, p. 380-396, 2006.

BACDAYAN, P. Quality improvement teams that stall due to poor project selection: an exploration of contributing factors. Total Quality Management, v. 12, n. 5, p. 589-598, 2001.

BAÑEGIL, T. El sistema JIT y la flexibilidad de la producción. Madrid: Pirâmide, 1993.

BARRICK, M.; ALEXANDER, R. A review of quality circle efficacy and the existence of positive-findings bias. Personnel Psychology, v. 40, n. 3, p. 579-592, 1987.

BODEK, N. Quick and Easy Kaizen. IIE Solutions, v. 34, n. 7, p. 43-45, 2002.

BOND, T. C. The role of performance measurement in continuous improvement. International Journal of Operations \& Production Management, v. 19, n. 12, p. 1318, 1999.

BUCH, K.; SPANGLER, R. The effects of quality circles on performance and promotions. Human relations, v. 43, n. 6, p. 573-582, 1990.

BUSHELL, S. Implementing plan, do, check and act. The Journal for Quality and Participation, v. 15, n. 5, p. 58-62, 1992.

CHOI, T. Y.; RUNGTUSANATHAM, M.; KIM, J. S. Continuous improvement on the shop floor: lessons from small to midsize firms. Business Horizons, v. 40, n. 6, p. 45-50, 1997.

DE LANGE-ROS, E.; BOER, H. Theory and practice of continuous improvement in shop-floor teams. International Journal Of Technology Management, v. 22, n. 4, p. 344-358, 2001.

DEMING, W. E. The New Economics: For Industry, Government, Education. Cambridge: MIT Center for Advanced Engineering Study, 1993.

DRACH, B. Use manufacturing standards to drive continuous cost improvement, Production and Inventory Management Journal, v. 35, n. 1, p. 20-25, 1994.

FABI, B.; PONS, O. Los circulos de calidad una experiencia internacional. Alta Dirección, n. 192, p. 275-285, 1995.

FAIRBANK, J. F.; WILLIAMS, S. D. Motivating Creativity and Enhancing Innovation through Employee Suggestion System Technology. Creativity \& Innovation Management, v. 10, n. 2, p. $68,2001$.
FRESE, M.; TENG, E.; WIJNEN, C. J. D. Helping to improve suggestion systems: Predictors of making suggestions in companies. Journal of Organizational Behavior, v. 20, n. 7, p. $1139,1999$.

GARCÍA LORENZO, A.; PRADO PRADO, J. C. Los sistemas de participación del personal en España. Diferencias en función del tamaño,certificación ISO 9000 y sector de actividad de las compañías. Alta Dirección, n. 220, p. 81-94, 2001.

GARCIA-LORENZO, A.; PRADO, J. C. Employee participation systems in Spain. Past, present and future. Total Quality Management \& Business Excellence, v. 14, n. 1, p. 15-24, 2003.

GREENBAUM, H.; KAPLAN, I.; METLAY, W. Evaluation of problem solving groups: the case of cuality circles programs. Group \& Organization Studies, v. 13, n. 2, p. 133-147, 1988.

GRIFFIN, R. Consequences of quality circles in an industrial setting: a longitudinal assessment. Academy of management Journal, v. 31, n. 2, p. 336-358, 1988.

GRÜTTER, A. W.; FIELD, J. M.; FAULL, N. H. B. Work team performance over time: three case studies of South African manufacturers. Journal of Operations Management, v. 20, n. 5, p. 641-657, 2002.

JACKSON, T.; DYER, C. Diagnóstico corporativo: una herramienta para alcanzar la excelência. 1 ed. Madrid: TGP Hoshin (PRODUCTIVITY PRESS), 1998.

KERRIN, M.; OLIVER, N. Collective and individual improvement activities: the role of reward systems. Personnel Review, v. 31, n. 3, p. 320-337, 2002.

LAWLER III, E. E. High involvement Management. San Francisco: Jossey-Bass, 1991.

LAWLER III, E. E.; MOHRMAN, S.; BENSON, G. Organizing for high performance: employee involvement, TQM, reengineering, and knowledge management in the fortune 1000. San Francisco: Jossey-Bass, 2001.

LI-PING, T.; TOLLISON, P.; WHITESIDE, H. Managers' attendance and the effectiveness of small work groups: the case of quality circles. The journal of Social Psychology, v. 13, n. 3 , p. $335-344,1988$.

LLOYD, G. C. Stuff the suggestions box. Total Quality Management, v. 10, n. 6, p. 869, 1999. 
MODARRESS, B.; ANSARI, A.; LOCKWOOD, D. L. Kaizen costing for lean manufacturing: a case study. International Journal of Production Research, v. 43, n. 9, p. 1751-1760, 2005.

NAIR, A. Meta-analysis of the relationship between quality management practices and firm performance--implications for quality management theory development. Journal of Operations Management, 2006. In Press.

PRADO PRADO, J. C. The implementation of continuous improvement through the participation of personnel: A case study. Production and Inventory Management Journal, v. 39, n. 2, p. 11, 1998.

PRADO, J. C. Beyond quality circles and improvement teams. Total Quality Management, v. 12, n. 6, p. 789-798, 2001.

RAPP, C.; EKLUND, J. Sustainable development of improvement activities: The long-term operation of a suggestion scheme in a Swedish company. Total Quality Management, v. 13, n. 7, p. 945-969, 2002.

SANCHEZ, J. I.; KRAUS, E.; WHITE, S.; WILLIAMS, M. Adopting high-involvement human resource practices. Group \& Organization Management, v. 24, n. 4, p. 461, 1999.

SCHONBERGER, R. J. World Class Manufacturing: the next decade. New York: Free Press, 1996.

SCHROEDER, R. G.; BATES, K. A.; JUNTTILA, M. A. A resourcebased view of manufacturing strategy and the relationship to manufacturing performance. Strategic Management Journal, v. 23, n. 2, p. 105, 2002.

SCHURING, R. W. Operational autonomy explains the value of group work in both lean and reflective production. International Journal of Operations \& Production Management, v. 16, n. 2, p. 171-182, 1996.
SCHURING, R. W.; LUIJTEN, H. Reinventing suggestion systems for continuous improvement. International Journal of Technology Management, v. 22, n. 4, p. 359-372, 2001.

SILlINCE, J. A. A.; SYKES, G. M. H.; SINGH, D. P. Implementation, problems, success and longevity of quality circle programmes: A study of 95 UK organizations. International Journal of Operations and Production Management, v. 16, n. 4, p. 88-111, 1996.

STOHL, C. Bridging the parallel organization: a study of quality circles effectivness. In: H. Mc Laughlin (Ed.). Communication yearbook. California: Sage, 1987, p. 416-430.

STOHL, C.; COOMBS, W. T. Cooperation or Cooptation: An Analysis of Quality Circle Training Manuals. Management Comunication Quarterly, v. 2, n. 1, p. 63-89, 1988.

TAPPING, D.; LUYSTER, T.; SHUKER, T. Value Stream management eight steps to planning, mapping, and sustaining lean improvements. 1 ed. New York: Productivity Press, 2002.

TERZIOVSKI, M.; SOHAL, A. S. The adoption of continuous improvement and innovation strategies in Australian manufacturing firms. Technovation, v. 20, n. 10, p. 539-550, 2000

VAN DIJK, C.; VAN DEN ENDE, J. Suggestion systems: transferring employee creativity into practicable ideas. R \& D Management, v. 32, n. 5, p. 387-395, 2002.

WOOD, A. Managing Employees' Ideas From Where do Ideas Come? Journal for Quality \& Participation, v. 26, n. 2, p. 22, 2003.

YIN, R. Case study research. 2 ed. sage: Ed. Thousand Oaks, 1994. 


\section{Anexo}

\section{Cálculo de ahorros de personas o tiempo de obreros}

Se calcula el tiempo o la cantidad de obreros necesarios para efectuar determinada tarea con el método o los instrumentos antiguos y, posteriormente, se calcula lo que se tarda en efectuar la tarea con el nuevo método o instrumentos. Hay que tener en cuenta el número de veces que se repiten las operaciones a lo largo del día y el número de obreros que intervienen directa o indirectamente en las operaciones para el cálculo del ahorro.

\section{Cálculo de ahorros de materiales o mate- rias primas}

Se calcula teniendo en cuenta, la cantidad de kilos que se producen al año del producto o de los productos a los que se efectúa la mejora y, el precio del producto y/o la repercusión que tiene la mejora en el coste del producto. Otras veces, el cálculo del ahorro en materias primas o materiales se debe al ahorro en el coste de las materias primas. Por ejemplo, si se puede utilizar un envase diferente para envolver un determinado producto porque las cualidades del envase permiten obtener los mismos beneficios de higiene y conservación a menor precio, este cálculo es inmediato si consideramos el ahorro en la materia prima y el número de envases utilizados en un año para producirse el producto.

\section{Cálculo de ahorro por ergonomía o lesio- nes en obreros}

Se calculan las posibles lesiones que suele haber acarreado el problema o la oportunidad de mejora y el efecto de la baja laboral sobre el ritmo normal de trabajo. Para estimar estos cálculos, se dispone de un programa informático creado y proporcionado por el sindicato U.G.T (Unión General de Trabajadores) y la mutua de accidentes. Por ejemplo, una persona que se cae accidentalmente y se rompe una pierna en la cámara de congelación, supone un coste de baja equivalente al coste del obrero más los costes de contratación y formación de una persona para el puesto vacante. Este tipo de cálculo no es exacto, puesto que los costes dependen de las personas y del puesto que ocupan, pero permite estimar la realidad con la aproximación suficiente.

\section{Cálculo de ahorros de mejor aprovecha- miento de máquinas}

Se calcula teniendo en cuenta la reducción del tiempo de ciclo de la máquina o de algún determinado parámetro como el consumo.

\section{Cálculo de ahorros de difícil cuantificación}

En determinadas ocasiones, existen cálculos de difícil estimación como pueden ser los ahorros por limpieza del lugar de trabajo, orden de la sala, mejora de calidad del producto. Para atender al cálculo de estos ahorros, se ha intentado siempre buscar algún criterio objetivo, aunque a veces se ha calculado subjetivamente.

\section{Sobre los autores}

\section{Juan A. Marin-García}

ROGLE. Departamento Organización de Empresas, Universidad Politécnica de Valencia, Edificio 7D, App. Correos: 22012, 46071, Valencia, Spain,

e-mail: jamarin@omp.upv.es

\section{Manuela Pardo-del-Val \\ Tomas Bonavia}

Universitat de València,

Avd. Blasco lbáñez, n 13. 46010, Valencia, España,

e-mails: manoli.pardo@uv.es; tomas.bonavia@uv.es

Agradecimientos: Agradecemos los comentarios realizados por los revisores anónimos, que nos han ayudado a mejorar nuestro artículo. 